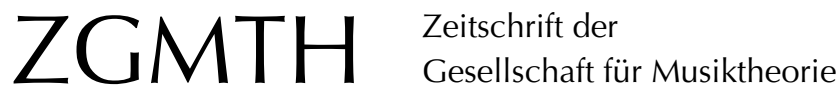

Polth, Michael (2011): Bibliographie zur Tonfeld-Analyse nach Albert Simon. ZGMTH 8/2, 365-367. https://doi.org/10.31751/648

(C) 2011 Michael Polth

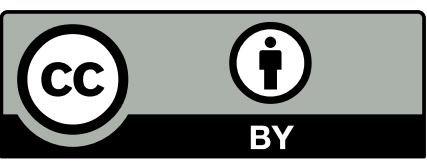

Dieser Text erscheint im Open Access und ist lizenziert unter einer Creative Commons Namensnennung 4.0 International Lizenz.

This is an open access article licensed under a

Creative Commons Attribution 4.0 International License.

veröffentlicht / first published: 17/11/2011

zuletzt geändert / last updated: 25/10/2013 


\title{
Bibliographie zur Tonfeld-Analyse nach Albert Simon
}

\author{
Michael Polth
}

Diese Bibliographie stellt alle bislang erschienenen oder in Vorbereitung befindlichen Schriften zusammen, in denen auf die Tonfeld-Analyse nach Albert Simon im weitesten Sinne Bezug genommen wird. Sie umfasst sowohl Beiträge, die sich unmittelbar mit der Anwendung, der Weiterentwicklung oder mit den methodischen Grundlagen der Analyse beschäftigen oder in diese einführen, als auch solche, die historische oder systematische Aspekte des Ansatzes oder das Verhältnis der Tonfeld-Analyse zu anderen Analysemethoden erörtern. Darüber hinaus enthält das Verzeichnis auch Texte, in denen die Tonfeld-Analyse als Mittel zur Beantwortung von Fragen dient, die anderen musiktheoretischen bzw. musikwissenschaftlichen Bereichen entstammen als der Tonfeld-Theorie selbst oder ihrem Umfeld.

Bodamer, Konstantin (2011), »Albert Simon - ein ungarischer Autor«, ZGMTH 8/2.

— (i. V.), „Tradition und Moderne bei Franz Liszt«, in: Blume, Jürgen und Konrad Georgi (Hg.), Musiktheorie und Improvisation. Kongressbericht der IX. Jahrestagung der Gesellschaft für Musiktheorie in Mainz, Mainz: Schott.

Haas, Bernhard (2004), Die neue Tonalität von Schubert bis Webern. Hören und Analysieren nach Albert Simon, Wilhelmshaven: Noetzel.

(2010), „Zur Sonatenform II mit analytischen Bemerkungen zum ersten Satz von Mozarts Sonate KV 570«, in: Funktionale Analyse. Musik - Malerei - Antike Literatur. Analyse Fonctionnelle. Musique - Peinture - Littérature classique. Kolloquium/ Colloque Paris, Stuttgart 2007, hg. von Bernhard Haas und Bruno Haas, Hildesheim u. a.: Olms, 261-294.

(2010), »R. W. - Venezia de Franz Liszt, avec une introduction à la théorie d'Albert Simon«, in: Funktionale Analyse. Musik - Malerei - Antike Literatur. Analyse Fonctionnelle. Musique - Peinture - Littérature classique. Kolloquium/Colloque Paris, Stuttgart 2007, hg. von Bernhard Haas und Bruno Haas, Hildesheim u. a.: Olms, 375-396.

(2011), "Zu zwei Bartók-Analysen von Albert Simon«, ZGMTH 8/2.

(i. V.), »Beobachtungen am Anfang von Max Regers Symphonischer Phantasie op. 57", in: Bericht über den VI. Kongress der GMTH in Weimar, hg. von Klaus Heiwolt, Hildesheim u. a.: Olms. 
- (i. V.), »Zur Sonatenform I«, in: Kongressbericht zum Internationalen Symposion zur Entwicklung der Kompositionstechnik bei W. A. Mozart Mannheim 2006.

(i. V.), »Analytische Fragmente zum ersten Satz von Bruckners VI. Symphonie«, in: Brahms und Bruckner, Musiktheoretisches Symposion Linz 2008, hg. von Christoph Hust.

(i. V.), „Über Mikrotonalität und Vieltönigkeit oder: wie die Musik von Bach bis Wagner das Hören und Denken der vielen Töne beeinflusst hat", in: Bericht über den Mikrotonalitätskongress Stuttgart 2011, hg. von Caspar Johannes Walter und Cordula Pätzoldt.

— (i. V.), »Improvisation, C. Ph. E. Bach, César Franck, and Albert Simon«, in: Bericht über das Improvisationssymposion Amsterdam 2011, hg. von Hans Fidom.

Hust, Christoph (2009), „Zur Verwendung musikalischer Idiome in Arthur Honeggers Choral für Orgel«, in: Kirchenmusikalisches Jahrbuch 93, hg. von Günther Massenkeil, Paderborn: Schöningh, 119-138.

Moss, Fabian C. (2011), „Albert Simons Theorie der Tonfelder und John Cloughs FlipFlop Circles im Vergleich«, http://www.scribd.com/doc/49350030/Albert-SimonsTheorie-der-Tonfelder-und-John-Cloughs-Flip-Flop-Circles-im-Vergleich

Nowak, Stefan (2011), „Schuberts frühe Sonatenform und die Tonfeldtheorie», ZGMTH $8 / 2$.

Polth, Michael (2006), »Tonalität der Tonfelder. Anmerkungen zu Bernhard Haas ১Die neue Tonalität von Schubert bis Webern. Hören und Analysieren nach Albert Simon««, ZGMTH 3/1, Hildesheim u. a.: Olms, 167-178.

(2006): »In den Freiräumen der Schenkerschen Tonalität: Harmonische Effekte durch Tonfelder in der Prager Sinfonie«, Dutch Journal of Music Theory 11/3, 151-163.

- (2007), „Zum Verhältnis zwischen Satztechnik und musikalischem Zusammenhang: Bemerkungen zu einer Sequenz in Bruckners 6. Symphonie«, in: `Vom Erkennen des Erkanntenr. Musikalische Analyse und Editionsphilologie. Festschrift für Christian Martin Schmidt, hg. von Thomas Ahrend, Heinz von Loesch und Frederike Wißmann, Wiesbaden u. a.: Breitkopf \& Härtel, 335-344.

(2007), "Satztechnische Modelle und moderne Tonalität. Zu einem Archaismus in Bruckners d-Moll-Messe«, in: Bruckner-Tag Mannheim 2006: Anton Bruckner - Die geistliche Musik (= Bruckner-Vorträge), hg. von Rainer Boss, Wien: Musikwissenschaftlicher Verlag, 47-64.

(2009), »Atonalität und musikalischer Zusammenhang in Weberns Orchesterstück op. 6 Nr. 3. Ein Beitrag zur Theorie der Tonfelder von Albert Simon«, Jahrbuch 2008|2009 des Staatlichen Instituts für Musikforschung Preußischer Kulturbesitz, Mainz u. a.: Schott, 87-121.

(2011), »Zur Artikulation von Tonfeldern bei Brahms, Debussy und Stockhausen«, ZGMTH 8/2.

Rohringer, Stefan (2009), »Tonalität in Franz Schuberts späten Sonatenformen. Überlegungen zum Kopfsatz des Klaviertrios B-Dur D 898«, ZGMTH 6/2-3, Hildesheim u. a.: Olms, 273-308. 
(2011), „Don Ottavio: Figur versus Medium. Zum Verhältnis von Prager und Wiener Fassung des >Don Giovanni««, Musik und Ästhetik 58, 5-32.

(i. V.), «Schenker, Luhmann und das Problem der ssteigenden Urlinie«», in: Schenkerian Analysis - Analyse nach Heinrich Schenker. Bericht über den internationalen Schenker-Kongreß in Berlin, Sauen und Mannheim, 4.-12. Juni 2004, hg. von Oliver Schwab-Felisch, Michael Polth und Hartmut Fladt, Hildesheim u. a.: Olms.

(i. V.), »Das Eigene im Fremden. Zu Mozarts Klaviersuite KV 399 (385i)«, in: Kongressbericht zum Internationalen Symposion zur Entwicklung der Kompositionstechnik bei W.A. Mozart Mannheim 2006, hg. von Michael Polth.

(i. V.), »Franz Schubert, die Wiener Generalbasslehre seiner Zeit und die historisch informierte Analyse«, in: Im Schatten des Kunstwerks - Komponisten als Theoretiker in Wien vom 17. Jahrhundert bis Anfang 19. Jahrhundert. Internationaler Kongress für Musiktheorie, Wien 3. bis 5. Mai 2007.

— (i. V.), »ohannes Brahms: Neun Lieder und Gesänge für eine Singstimme und Klavier op. 32«, in: Johannes Brahms. Interpretationen seiner Werke, hg. von Claus Bockmaier und Siegfried Mauser.

_ (i. V.), »Aspekte der Funktionalität in Johannes Brahms' Intermezzo h-moll op. $119,1 \ll, Z G M T H, 9 / 2$.

Schild, Johannes (2010), »»... zum Raum wird hier die Zeit.ı Tonfelder in Wagners Parsifal«, in: Funktionale Analyse. Musik - Malerei - Antike Literatur. Analyse Fonctionnelle. Musique - Peinture - Littérature classique. Kolloquium/Colloque Paris, Stuttgart 2007, hg. von Bernhard Haas und Bruno Haas, Hildesheim u. a.: Olms, 313-373.

Schiltknecht, Dres (2011), » Konstruktı und `Funktion`. Eine Herleitung der Simonschen Tonfelder«, ZGMTH 8/2.

Simon, Albert (1983), „Béla Bartók: ‘Secondes mineures - septièmes majeures` (Mikrokosmos, VI/144)«, in: Schweizerische Musikzeitung/Revue musicale suisse 123 (1983), 82-86.

Sotirianos, Markus (2011), »Tonfelder« und traditionelle Tonalität. Beobachtungen zu Schuberts Lied Der Atlas", ZGMTH 8/2. 